\author{
John M. Budd \\ University of Missouri \\ USA
}

\title{
Whither Physics? Publication Data and the Future of Research in Physics
}

\begin{abstract}
The discipline of physics is an extraordinarily lively one, producing a huge literature. Since physics, as is the case with any science, is based on the answers to questions, it is a worthwhile endeavor to explore what fundamental areas are being studied. The exploration of what is published is valuable in its own right, since it helps to define what types of questions are being asked and the extent to which the literature reflects the questioning. Beyond that purpose, there is something of an ethical nature to the exploration. That ethical basis could, if one has sufficient hubris, be expressed as, what should the research field be. The proposed paper examines the publication landscape of sub-fields in physics and investigates the possibilities inherent in the largest body of literature - string theory.
\end{abstract}

Science is founded upon questions; scientists of all stripes ask and attempt to answer questions that are meaningful to humankind. The questions asked carry import; they are indicators of where scientists place import and how they are addressing that import. Special attention will be paid here to physics and its various sub-fields (active at the present moment). There are two reasons why the literature of physics is investigated: (1) revelation of the amount of data (defined here as numbers of published works) that is devoted to each of the sub-fields so that one can glean activity in the discipline of physics, and (2) depiction of some claims related to the most active of the sub-fields, along with the inference of an informational ethics associated with that sub-field. As is evident in Table 1, the most active sub-field now, and for a few decades, is that of string theory (frequently referred to as superstring theory).

Background is needed to paint a picture of the sub-field of string theory. The activity, in terms of publication, has a rather substantial history. As Steven Gubser (2010) points out, the beginnings came with some modest theorizing around 1968. He says that the first wave of substantive interest in the concept came in 1984 when "it was shown that string theory was free of anomalies" (p. 2). There are some who would dispute the claim of an anomaly-free theory, though, as we will soon see. Even so strong a proponent of string theory as Brian Greene (2003) prefers to temper his words: "string theory should be viewed as a work in progress whose partial completion has already revealed astonishing insights into the nature of space, time, and matter" and "In the final analysis, though, nothing is a substitute for definitive, testable predictions that can determine whether string theory has truly lifted the veil of mystery hiding the deepest truths of our universe" (p. 18). Lawrence Krauss (2012) may perhaps go furthest by saying that physicists have already proved why there is something rather than nothing in the universe. 
While Greene (2003) offers caveats, he does say, "String theory offers a novel and profound modification to our theoretical description of the ultramicroscopic properties of the universe - a modification that, physicists slowly realized, alters Einstein's general relativity in just the right way to make it fully compatible with the laws of quantum mechanics" (p. 136). It becomes necessary to examine the claims made by adherents to string theory, and that analysis will follow the presentation of Table 1. The source of the data used here is the database Scopus ${ }^{\circledR}$, searched on 3, October, 2017. The table represents the name of the subfield (and it is not suggested that these are all possible subfields) and the total number of published works included in Scopus.

Table 1

Publication Data of Physics Sub-Fields - Scopus

Quantum Gravity

15,023

Dark Energy

35,731

Dark Matter

36,965

Supersymmetry

37,515

Relativity

39,248

Neutrinos

42,238

Black Holes

47,093

$>500$ per year, 1995-present

Quantum Field Theory

81,107

Quantum Mechanics

109,492 


\section{String Theory (includes Superstrings)}

195,765

As is obvious from Table 1, the single largest subfield, in terms of quantity of published works, is string theory, even outpacing the much more mature area of quantum mechanics. This presentation does not deal with string theory as such (that is, it does not delve into the mathematics or theoretical subtleties). Rather, the examination is of the efficacy of the theory as a viable scientific enterprise and, more importantly, the ethical underpinnings of engagement in such a problematic theoretical premise. There will be some criticisms of the theory, but these will direct the investigation to the ethical matters presented by such a major scientific undertaking.

A similar search has been conducted in the Web of Science Core Collection ${ }^{\circledR}$, on 10 March 2018. Items were searched using the "topic" delineator. This search is intended to accomplish a check on the Scopus search. The results are presented in Table 2.

Table 2

Publication Data of Physics Sub-Fields - Web of Science Core Collection

Quantum Field Theory

11,156

Quantum Gravity

11,926

Dark Energy

14,104

Supersymmetry

23,596

Relativity

33,640

Dark Matter

40.776

Neutrinos

41,036

Black Holes

59,219

Quantum Mechanics 
64,088

\section{String or Superstring Theory}

137,069

The rankings in the two databases are not identical, but a pattern-especially the indication that string and superstring theory-becomes clear.

Krauss (2012), whose broad claim was mentioned earlier, says about string theory that, "We still have no idea is this remarkable edifice actually has anything to do with the real world" (p. 130). What he is critiquing is the putative existence of something like a Kuhnian paradigm (more will be said about Thomas Kuhn shortly). Krauss's criticism is echoed by no less a luminary than Roger Penrose (2016), who says, "there appears to be no results whatever that provide [string theory] with experimental support" (p. 3). Penrose (2016) does not invoke Kuhn, but he writes, An anomaly is something which occurs when a classically described theory. . . loses some key property when the rules of quantum mechanics are applied to it, usually a symmetry of some kind" (p. 37). These are damning statements, but they are not the harshest criticisms.

Peter Woit (2006) devotes an entire book to the problems of string theory and concludes, "No matter how things turn out, the story of superstring theory is an episode with no real parallel in the history of modern physical science. More than twenty years [plus the additional decade since his book was published] of intensive research by thousands of the best scientists in the world producing tens of thousands of scientific papers has not led to a single testable experimental prediction of the theory" (p. 203). Woit is joined by Lee Smolin (2006), who asserts, Lacking both fundamental principles and the mathematical formulation, we cannot say that we even know what string theory asserts" (p. xv). Together, Woit and Smolin paint a bleak picture.

This brings us back to Kuhn (1970), who offers what I will call an inchoate ethics. Kuhn's sociology describes what is occurring in the sub-field of string theory; a normal science is governed by a paradigm, which may or may not be concerned with the questions (or answers) of the field (pp. 46 ff.). Kuhn (1970) states, "Paradigms provide all phenomena except anomalies with a theory-determined place in the scientist's field of vision" (p. 97). See Penrose (above) for an appropriate corollary to Kuhn's observation. Perhaps most fittingly, Kuhn (1970) claims, "A paradigm governs, in the first instance, not a subject matter but rather a group of practitioners. Any study of paradigm-directed. . . research must begin by locating the responsible group or groups" (p. 180). Smolin (2006) is the only one of the mentioned critics of string theory who cites Kuhn (1970). Smolin notes that "method" is of secondary importance to the paradigm, thus solidifying the community's hold on normal science. And thus creating an ethically fraught predicament for the scientists. The presentation will go into more depth on Kuhn's (1970) observations; for example, he says, "Claims [in opposition to a governing 
paradigm] are particularly likely to succeed if the new paradigm displays a quantitative precision strikingly better than its older competitor" (pp. 153-54). Kuhn refers to the work of normal science as puzzle solving, but string theory appears to be unable to solve the most fundamental puzzles. Yet it persists in what can be seen as, at best, an ethical middle ground - stubborn refusal to admit defeat and absence of consequential success, buoyed only by a hope that difficulties will be resolved eventually. Meanwhile, an abundance of resources are poured into what has been, for decades, a dead end. The presentation will explore the ethical ramifications of the lack of results.

\section{References}

Greene, Brian. (2003). The Elegant Universe. New York: W. W. Norton

Gubser, Steven S. (2010). The Little Book of String Theory. Princeton: Princeton University Press.

Horgan, John. (2015). The End of Science. New York: Basic Books.

Krauss, Lawrence M. (2012). A Universe from Nothing. New York: Atia Paperback.

Kuhn, Thomas S. (1970). The Structure of Scientific Revolutions, $2^{\text {nd }}$ ed. Chicago: University of Chicago Press.

Penrose, Roger. (2016). Fashion, Faith, and Fantasy in the New Physics of the Universe. Princeton: Princeton University Press.

Smolin, Lee. (2006). The Trouble with Physics. New York: Houghton Mifflin.

Woit, Peter. (2006). Not Even Wrong. New York: Basic Books. 